\title{
Improving the N-terminal diversity of sansanmycin through mutasynthesis
}

\author{
Yuanyuan Shi, Zhibo Jiang, Xuan Lei, Ningning Zhang, Qiang Cai, Qinglian Li, Lifei Wang, Shuyi Si, Yunying Xie* \\ and Bin Hong*
}

\begin{abstract}
Background: Sansanmycins are uridyl peptide antibiotics (UPAs), which are inhibitors of translocase I (MraY) and block the bacterial cell wall biosynthesis. They have good antibacterial activity against Pseudomonas aeruginosa and Mycobacterium tuberculosis strains. The biosynthetic gene cluster of sansanmycins has been characterized and the main biosynthetic pathway elucidated according to that of pacidamycins which were catalyzed by nonribosomal peptide synthetases (NRPSs). Sananmycin A is the major compound of Streptomyces sp. SS (wild type strain) and it bears a non-proteinogenic amino acid, meta-tyrosine ( $m$-Tyr), at the N-terminus of tetrapeptide chain.
\end{abstract}

Results: ssaX deletion mutant SS/XKO was constructed by the $\lambda$-RED mediated PCR targeting method and confirmed by PCR and southern blot. The disruption of ssaX completely abolished the production of sansanmycin A. Complementation in vivo and in vitro could both recover the production of sansanmycin A, and the overexpression of SsaX apparently increased the production of sansanmycin A by $20 \%$. Six new compounds were identified in the fermentation culture of ssaX deletion mutant. Some more novel sansanmycin analogues were obtained by mutasynthesis, and totally ten sansanmycin analogues, MX-1 to MX-10, were purified and identified by electrospray ionization mass spectrometry (ESI-MS) and nuclear magnetic resonance (NMR). The bioassay of these sansanmycin analogues showed that sansanmycin MX-1, MX-2, MX-4, MX-6 and MX-7 exhibited comparable potency to sansanmycin A against $M$. tuberculosis $\mathrm{H}_{37} \mathrm{Rv}$, as well as multi-drug-resistant (MDR) and extensive-drug-resistant (XDR) strains. Moreover, sansanmycin MX-2 and MX-4 displayed much better stability than sansanmycin A.

Conclusions: We demonstrated that SsaX is responsible for the biosynthesis of $m$-Tyr in vivo by gene deletion and complementation. About twenty novel sansanmycin analogues were obtained by mutasynthesis in ssaX deletion mutant SS/XKO and ten of them were purified and structurally identified. Among them, MX-2 and MX-4 showed promising anti-MDR and anti-XDR tuberculosis activity and greater stability than sansanmycin A. These results indicated that ssaX deletion mutant SS/XKO was a suitable host to expand the diversity of the N-terminus of UPAs, with potential to yield more novel compounds with improved activity and/or other properties.

Keywords: $m$-Tyr, ssaX deletion mutant, Mutasynthesis, Novel sansanmycin analogues

\section{Background}

Sansanmycins [1], produced by Streptomyces sp. SS, belong to the uridyl peptide antibiotics (UPAs) including pacidamycins [2], napsamycins [3] and mureidomycins

\footnotetext{
*Correspondence: xieyy@imb.pumc.edu.cn; binhong69@hotmail.com; hongbin@imb.pumc.edu.cn

The Key Laboratory of Biotechnology of Antibiotics of Ministry of Health, Institute of Medicinal Biotechnology, Chinese Academy of Medical Sciences \& Peking Union Medical College, No.1 Tiantan Xili, Beijing 100050, China
}

[4]. They hold a common and unique structure (Fig. 1), a 3'-deoxyuridyl attached to a pseudo-tetra/pentapeptidyl backbone via an exocyclic enamide. The peptidyl chain exhibited interesting double reversals due to the $\beta$-peptidation of the $\mathrm{N}$-methyl-2,3-diaminobutyric acid (DABA) and a ureido linkage [5]. Sansanmycins exhibit good antibacterial activity against highly refractory pathogens including Pseudomonas aeruginosa and Mycobacterium tuberculosis [6]. With 1.5 million people killed by tuberculosis (TB) in 2014, the disease ranks 


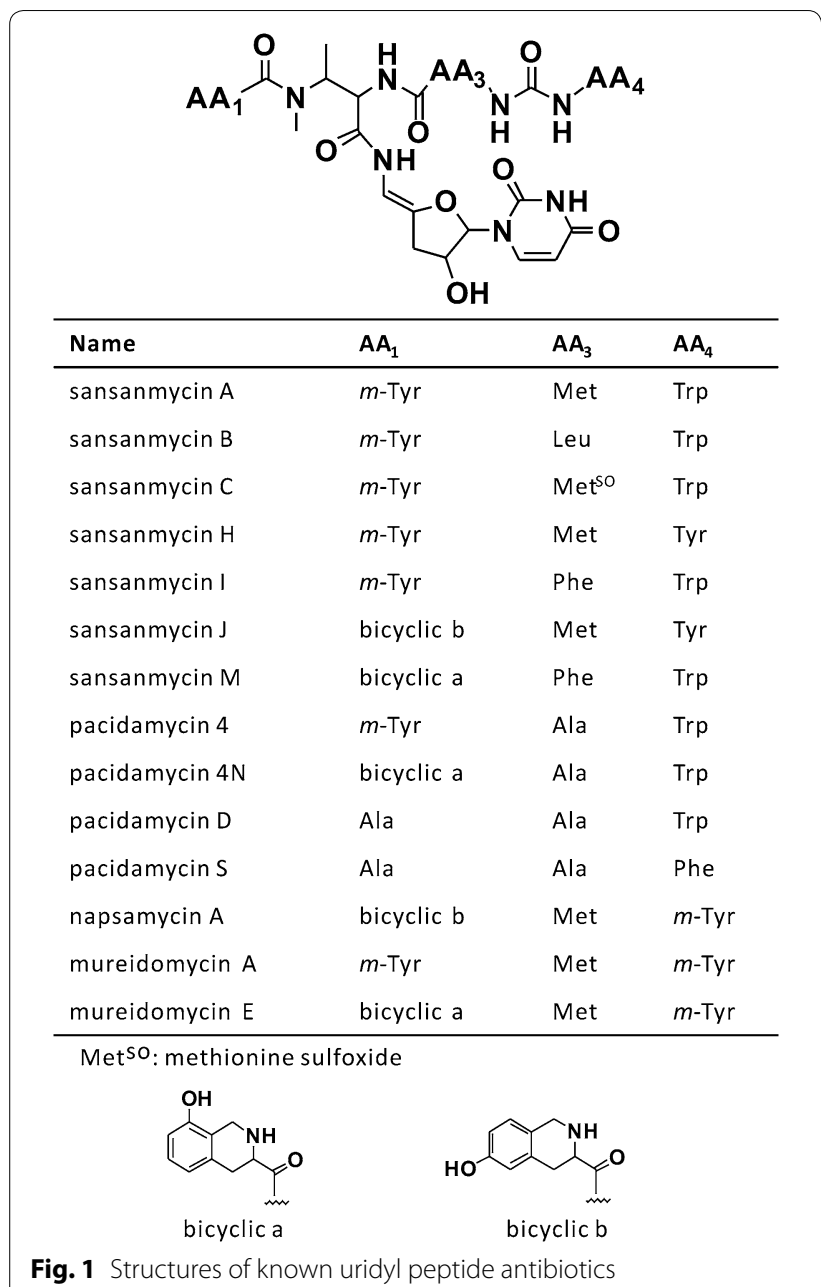

alongside human immunodeficiency virus as a leading killer worldwide [7]. The increasing emergence of multi-drug-resistant (MDR) and extensive-drug-resistant (XDR) tuberculosis make the treatment more difficult. So there is an urgent need to develop novel anti-TB drugs with no cross-resistance to current clinically used antibiotics. Sansanmycins and other UPAs are of interest, due that they inhibit a clinically unexploited target MraY (phospho-MurNAc-pentapeptide translocase, also known as translocase I) [8], which catalyzes the transfer of UDPMurNAc-L-Ala- $\gamma$-D-Glu- $m$-DAP-D-Ala-D-Ala onto lipid carrier undecaprenyl phosphate to give lipid intermediate I in the bacterial peptidoglycan biosynthetic pathway. The potential bioactivity against $M$. tuberculosis coupled to its intriguing structure made this natural product a fascinating anti-TB lead compound.

Recently, the biosynthetic gene clusters for pacidamycins [5, 9], napsamycins [10], and sansanmycins [11] were identified and characterized, indicating that the assembly of the pseudo-tetrapeptide chain is catalyzed by nonribosomal peptide synthetases (NRPSs) with highly dissociated modules [12]. Besides, the biosynthesis of uridyl pentapeptide of pacidamycins was catalyzed by the tRNA-dependent aminoacyltransferase $\mathrm{PacB}$, which transferred the alanyl residue from alanyl-tRNA to the $\mathrm{N}$-terminus of the pseudo-tetrapeptide [13]. In contrast to ribosomal peptide synthesis, non-ribosomally assembled peptides contain not only the 20 proteinogenic amino acids but also many different building blocks, such as DABA, D-amino acids, hydroxyl amino acids, $\mathrm{N}$ - and $\mathrm{C}$-methylated amino acids etc. Among them, non-proteinogenic amino acid meta-tyrosine ( $m$-Tyr) is rarely found in bacterial secondary metabolites. UPAs and a potent cyclophilin inhibitor sanglifehrin A (SFA) are two examples containing $m$-Tyr as one of the building blocks. SfaA, identified in the SFA biosynthetic gene cluster, was speculated to catalyze the biosynthesis of $m$-Tyr [14]. As a homologue of SfaA in pacidamycin biosynthetic gene cluster, PacX was characterized as a phenylalanine 3-hydroxylase that catalyzed the synthesis of $m$-Tyr from L-phenylalanine (L-Phe) in vitro [15]. In sansanmycin biosynthetic gene cluster, SsaX is homologous to PacX with amino acid identity of $80 \%$ across the whole protein, indicating that it is responsible for the biosynthesis of $m$-Tyr in Streptomyces sp. SS.

Although natural UPAs have potential to treat refractory infections, there is no UPAs entering clinical trials until now mainly due to their relatively poor physicochemical property. In previous studies, the N-terminal amino acid of the tetrapeptide of UPAs was supposed to be important functional group for the inhibition of MraY $[16,17]$. It was proposed that the protonated ammonium ion binds in place of the $\mathrm{Mg}^{2+}$ cofactor at the MraY active site via cis-amide linkage [17]. The $\mathrm{N}$-terminal amino acid of known UPAs is almost dominated by $m$-Tyr or different bicyclic acids (possibly derived from $m$-Tyr), except that some pacidamycins possess Ala instead (Fig. 1). In this study, we focus on the substitution of the N-terminal amino acid to get novel sansanmycin analogues by mutasynthesis. Mutasynthesis is a useful method in the generation of new antibiotic derivatives [18]. This approach could expand the chemical diversity of secondary metabolites and produce novel compounds with improved physicochemical properties or altered bioactivity. For example, it has been successfully employed to get novel nucleoside antibiotics such as nikkomycin analogues [19] and new ansamycin derivatives [20]. However, mutational biosynthesis has not been employed to obtain UPA derivatives so far.

Here, we demonstrated that SsaX is responsible for the biosynthesis of $m$-Tyr in vivo by gene deletion and complementation and the sansanmycin production could be increased through the overexpression of ssaX. Six new 
sansanmycin analogues were purified and characterized in $s s a X$ deletion mutant, indicating the substrate flexibility of the responsible NRPS. To expand the diversity of sansanmycins by mutasynthesis, different types of substrates were fed to the ssaX deletion mutant and some novel sansanmycin derivatives were obtained. These compounds were purified and structurally identified, some of which exhibited improved antibacterial activity or stability.

\section{Results}

\section{In-frame deletion of ssaX and its complementation}

In order to investigate the contribution of $s s a X$ to sansanmycin biosynthesis, an $s s a X$ deletion mutant SS/XKO was constructed from Streptomyces sp. SS by PCR targeting [21] using cosmid 13R-1 [11] which contains ssaX and the majority of other biosynthetic genes. Cosmid 13R-1SCP2KO was firstly constructed from cosmid 13R-1 with the minimal replicon of $\mathrm{SCP} 2 *$ replaced by ampicillin resistance gene in order to promote homologous recombination for the disruption of ssaX. Then the ssaX gene in 13R-1-SCP2KO was in-frame deleted and the resulted 13R-1-SCP2KO-XKO was introduced into the wild type strain by conjugation (Fig. 2a). The $s s a X$ in-frame deletion mutant SS/XKO was verified by PCR using primers PT-X-7 and PT-X-8 (Fig. 2a) and further confirmed by southern blot analysis (Fig. 2b, c). The coding region of ssaX was cloned into a pSET152 [22] -derived expression plasmid, pL646 [23], under the control of a strong constitutive promoter, $e r m E^{*}$ p , to give $\mathrm{pL}$-ssaX. The plasmid was introduced into SS/XKO strain by conjugation and the complementary strain $\mathrm{SS} / \mathrm{XKO} / \mathrm{pL}$-ssaX was resulted to exclude the potential polar effect in SS/XKO. Metabolites of constructed strains were scrutinized to compare with that of the wild type strain by HPLC, LC/MS and bioassay analysis. The major product in the wild type strain, sansanmycin A with $m$-Tyr at the $\mathrm{N}$-terminus of tetrapeptide chain, was not detected in SS/XKO (Fig. 2d). The culture broth of SS/XKO showed no antibacterial activity against $P$. aeruginosa 11 , which was used as the test microorganism for the bioassay of sansanmycins (Fig. 2e). Meanwhile, the complementary strain SS/ $\mathrm{XKO} / \mathrm{pL}$-ssaX completely recovered the production of sansanmycin A detected by HPLC analysis (Fig. 2d) and antibacterial bioassay (Fig. 2e).

In addition to genetic complementation in vivo, a chemical complementation by feeding the substrate $m$-Tyr $(3 \mathrm{mM})$ to the fermentation medium of the $s s a X$ deletion mutant SS/XKO was also performed. The result of HPLC showed that the major product of this culture broth was sansanmycin A and the production level was significantly higher than the wild type strain (Fig. 3a). Consistent with this result, the inhibition zone was bigger than that of wild type strain in the bioassay (Fig. 3b). All of these results demonstrated that SsaX was responsible for the biosynthesis of $m$-Tyr in sansanmycin biosynthetic pathway.

To further investigate the role of $s s a X$ in sansanmycin biosynthesis, the plasmid pL-ssaX was transferred into the wild type strain by conjugation to give the $s s a X$ overexpression strain SS/pL-ssaX, with pSET152 transferred strain SS/pSET152 as a control. With the same growth curves, the overexpression of SsaX apparently increased the production of sansanmycin A by $20 \%$ from HPLC analysis (Fig. $2 \mathrm{~d}$ ). The antibacterial activity against $P$. aeruginosa 11 also showed that SS/pL-ssaX exhibited bigger inhibition zone than the control strain (Fig. 2e). This result, together with the result of chemical complementation, suggested that the biosynthesis of $m$-Tyr catalyzed by SsaX is at least one of the rate-limiting steps in the sansanmycin production [24].

\section{Isolation and structural determination of sansanmycin analogues in ssaX deletion mutant}

Through the HPLC and LC/MS analysis, a series of minor components of sansanmycin analogues were detected in the cultivated broth of ssaX deletion mutant SS/XKO. In order to characterize these compounds, SS/XKO fermentations were scaled up to obtain enough amount of material for further analysis. The target compounds were enriched by macroporous absorbant resin from fermentation broth, then isolated using DEAE-Sephadex A25 guided by HPLC-UV to yield the crude sansanmycin analogues. Subsequently, the crude compounds were purified by preparative HPLC. As a result, six new sansanmycin analogues were obtained and designated as

\footnotetext{
(See figure on next page.)

Fig. 2 Effects of the deletion of ssaX on sansanmycin production. a Construction of the ssaX deletion mutant (SS/XKO) by PCR targeting and its PCR verification (using primers PT-X-7 and PT-X-8). Primers PT-X-1 and PT-X-2 were used to amplify a streptomycin resistance cassette (aadA gene), which substituted ssaX on cosmid 13R-1-SCP2KO. bla, ampicillin resistance marker; aac(3)IV, apramycin resistance marker. b, c Southern blot hybridization of wild type strain and SS/XKO. The genomic DNAs were digested with BamHI and hybridized with specific probes of ssaX deleted fragment (b) and the fragment downstream of ssaX (c) respectively. The wild type strain showed the hybridized band of $4.4 \mathrm{~kb}$ both in $\mathbf{b}$ and $\mathbf{c}$. The correct ssaX deletion mutant showed no hybridized band in $\mathbf{b}$ and the hybridized band of $3.7 \mathrm{~kb}$ instead of $4.4 \mathrm{~kb}$ in $\mathbf{c}$. $\mathbf{d}$ HPLC analysis of different strains. Sansanmycin $\mathrm{A}$ is the major compound in wild type strain with $m$-Tyr at N-terminus. e Antibacterial activity analysis of different strains. 1, wild type strain; 2, SS/XKO; 3, SS/XKO/pL-ssaX; 4, SS/pL-ssaX
} 
a

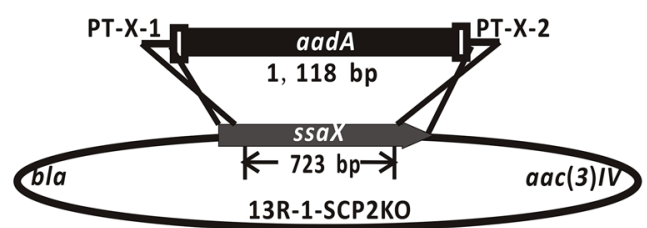

13R-1-SCP2KO

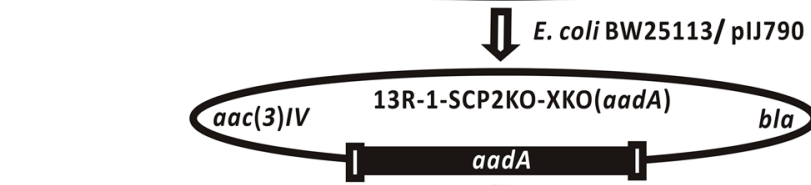

I E. coli DH5 $\alpha /$ BT340

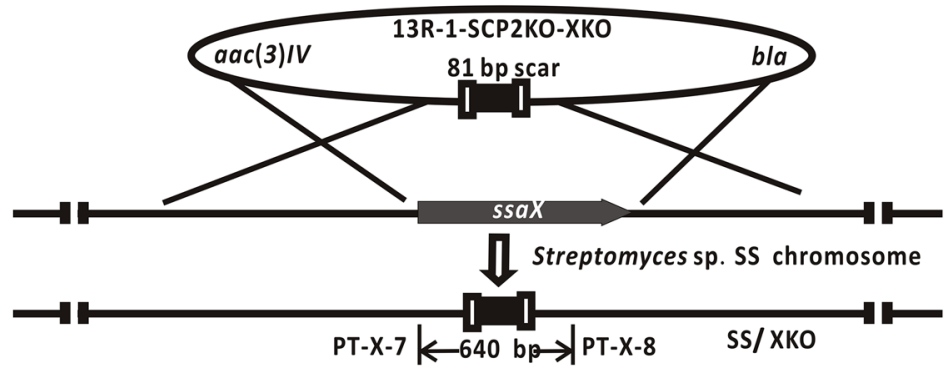

b

C

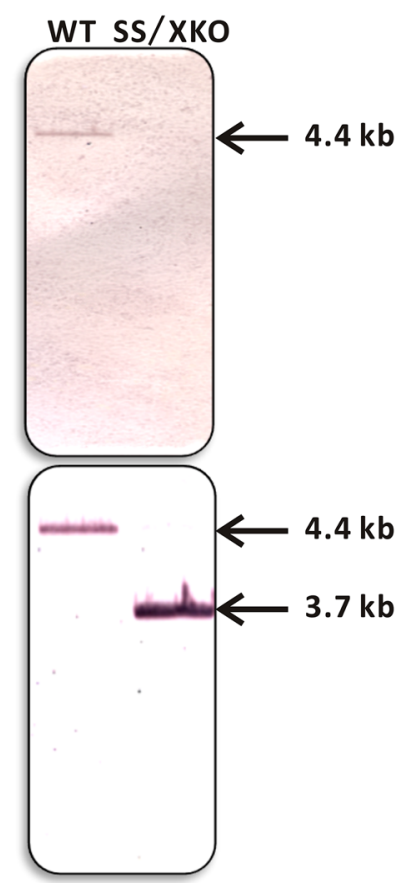

d

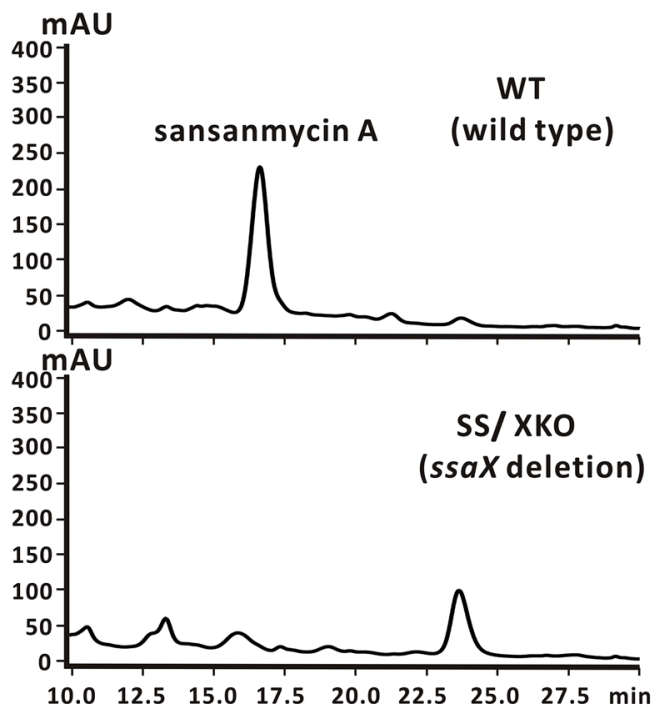

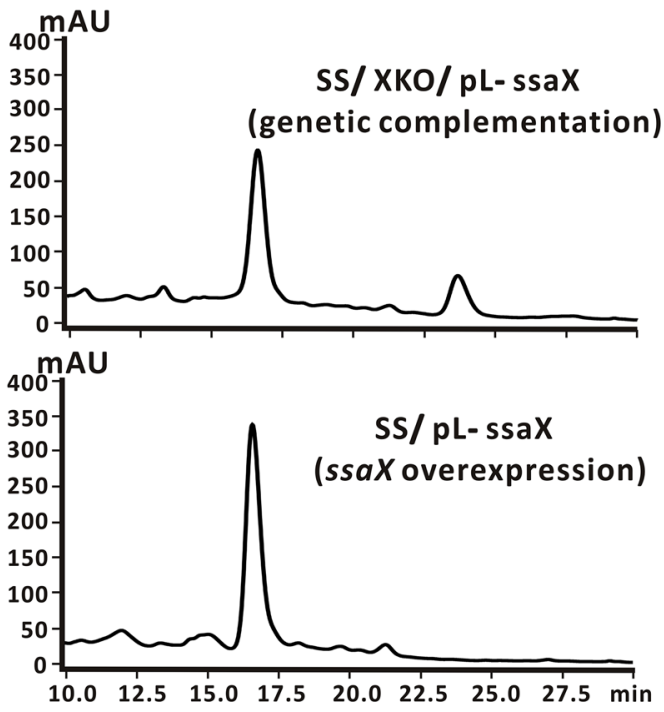

e

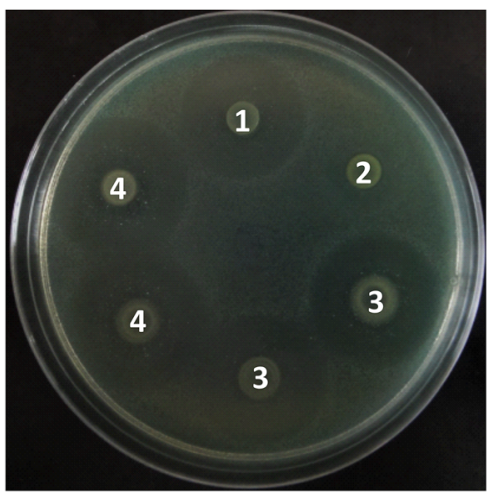




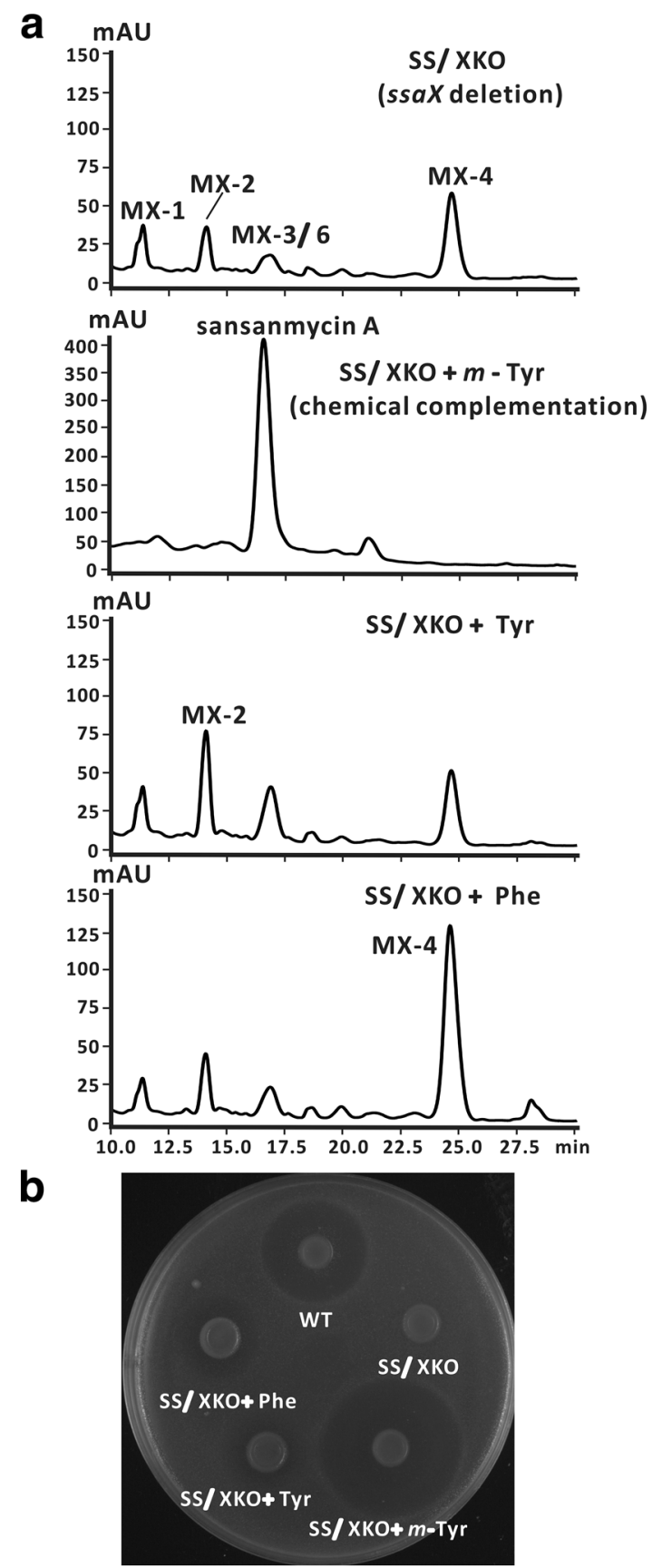

Fig. 3 Effects of supplying SS/XKO with meta-tyrosine ( $m$-Tyr), tyrosine (Tyr) and phenylalanine (Phe). a HPLC analysis of the culture broth of different supplementations. The final concentration of each exogenous substrate was $3 \mathrm{mM}$. b Antibacterial activity analysis of the culture broth of different supplementations

sansanmycin MX-1 to MX-6 respectively. Their structures were elucidated by electrospray ionization mass spectrometry (ESI-MS), ESI-MS/MS and nuclear magnetic resonance (NMR) spectroscopic analysis.
Sansamycin MX-1, MX-2, MX-4 and MX-6 have molecular weights of 700, 863, 847 and 831, respectively. The ESI-MS/MS results of them (Fig. 4) showed the same fragment patterns with sansanmycin A. All of these four compounds showed the same diagnostic fragment with sansanmycin $\mathrm{A}, \mathrm{m} / \mathrm{z} 701$, corresponding to the loss of the $\mathrm{N}$-terminal amino acid $\left(\mathrm{AA}_{1}\right)$, suggesting they varied at the $\mathrm{N}$-terminal amino acid. In support of this hypothesis, the ${ }^{1} \mathrm{H}$ NMR data for MX-1 (Fig. 5a; Additional file 1: Table S1), MX-2 (Additional file 1: Table S2, Figure S4), MX-4 (Additional file 1: Table S3, Figure S6) and MX-6 (Additional file 1: Table S4, Figure S8) proved to be very similar to that of sansanmycin A, with differences limited to replacement of the N-terminal $m$-Tyr in sansanmycin A $[\delta 7.23(\mathrm{t}, 1 \mathrm{H}), 6.78(\mathrm{~d}, 1 \mathrm{H}), 6.75(\mathrm{~d}, 1 \mathrm{H}), 6.72(\mathrm{~s}$, $1 \mathrm{H}), 4.04(\mathrm{~m}, 1 \mathrm{H}), 2.51(\mathrm{~m}, 1 \mathrm{H}), 2.89(\mathrm{~m}, 1 \mathrm{H})]$ [1] with a hydrogen in MX-1 $(\delta 10.78)$, a tyrosine (Tyr) in MX-2 [ $\delta$ $7.51(\mathrm{~d}, 2 \mathrm{H}), 6.97(\mathrm{~d}, 2 \mathrm{H}), 4.15(\mathrm{~m}, 1 \mathrm{H}), 3.08(\mathrm{~m}, 1 \mathrm{H}), 2.97$

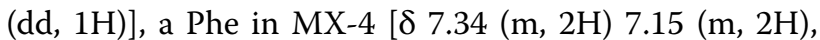
$7.27(\mathrm{~m}, 1 \mathrm{H}), 3.92(\mathrm{~m}, 1 \mathrm{H}), 2.73(\mathrm{~m}, 1 \mathrm{H}), 2.86(\mathrm{~m}, 1 \mathrm{H})]$ and a methionine (Met) in MX-6 $[\delta 4.31(\mathrm{~m}, 1 \mathrm{H}), 2.47(\mathrm{~m}$, $1 \mathrm{H}), 2.37(\mathrm{~m}, 1 \mathrm{H}), 1.99(\mathrm{~s}, 3 \mathrm{H}), 1.81(\mathrm{~m}, 1 \mathrm{H})]$. Interpretation of the ${ }^{13} \mathrm{C}$ (Fig. 5b; Additional file 1: Figure S5, S7, S9) and 2D NMR data (Fig. 5c; Additional file 1: Figures S1-S3) also confirmed these proposed structures.

MX-3 has a molecular weight of 824, 16 mass units smaller than that of sansanmycin $\mathrm{H}$ [25], attributed to the loss of an oxygen atom. Furthermore, the ESI-MS/ MS spectrum of MX-3 also showed the same diagnostic fragment with sansanmycin $\mathrm{H}, \mathrm{m} / \mathrm{z} 678$ for the loss of the N-terminal $m$-Tyr, which suggested that the $m$-Tyr in sansanmycin $\mathrm{H}$ was replaced by a Phe in MX-3. Comparison with that of sansanmycin $\mathrm{H}$, the ${ }^{1} \mathrm{H}$ NMR spectrum of MX-3 (Additional file 1: Figure S10) showed a different aromatic pattern from sansanmycin $\mathrm{H}$, with a Phe $[\delta 7.15$ $(\mathrm{m}, 2 \mathrm{H}), 7.15(\mathrm{~m}, 2 \mathrm{H}), 7.27(\mathrm{~m}, 1 \mathrm{H})]$ instead of $m$-Tyr $[\delta$ $7.23(\mathrm{t}, 1 \mathrm{H}), 6.78(\mathrm{~d}, 1 \mathrm{H}), 6.75(\mathrm{~d}, 1 \mathrm{H}), 6.72(\mathrm{~s}, 1 \mathrm{H})]$ [25].

MX-5 has a molecular weight of 863,16 mass units greater than MX-4, corresponding to an extra oxygen atom. Comparison with that of MX-4, the ${ }^{1} \mathrm{H}$ NMR spectrum of MX-5 (Additional file 1: Figure S11) showed a downfield shifted methyl proton signal [from $\delta 2.01$ ($\left.\mathrm{SCH}_{3}\right)$ to $\left.2.46\left(-\mathrm{SOCH}_{3}\right)\right]$, which hinted the oxidation of MX-4 to MX-5. The ESI-MS/MS analysis (Fig. 4) further confirmed this hypothesis.

The obtained six compounds MX-1-6 were new members of sansanmycin family. Compared with SS-A, MX-1, short of the $\mathrm{N}$-terminal $m$-Tyr, bears a tri-pseudopeptide backbone that was found in the family of UPAs for the first time. It is the accumulated precursor when there is no $m$-Tyr present in SS/XKO, which is also the obvious evidence that SsaX catalyzes the biosynthesis of $m$ Tyr. The other five compounds were different from the 


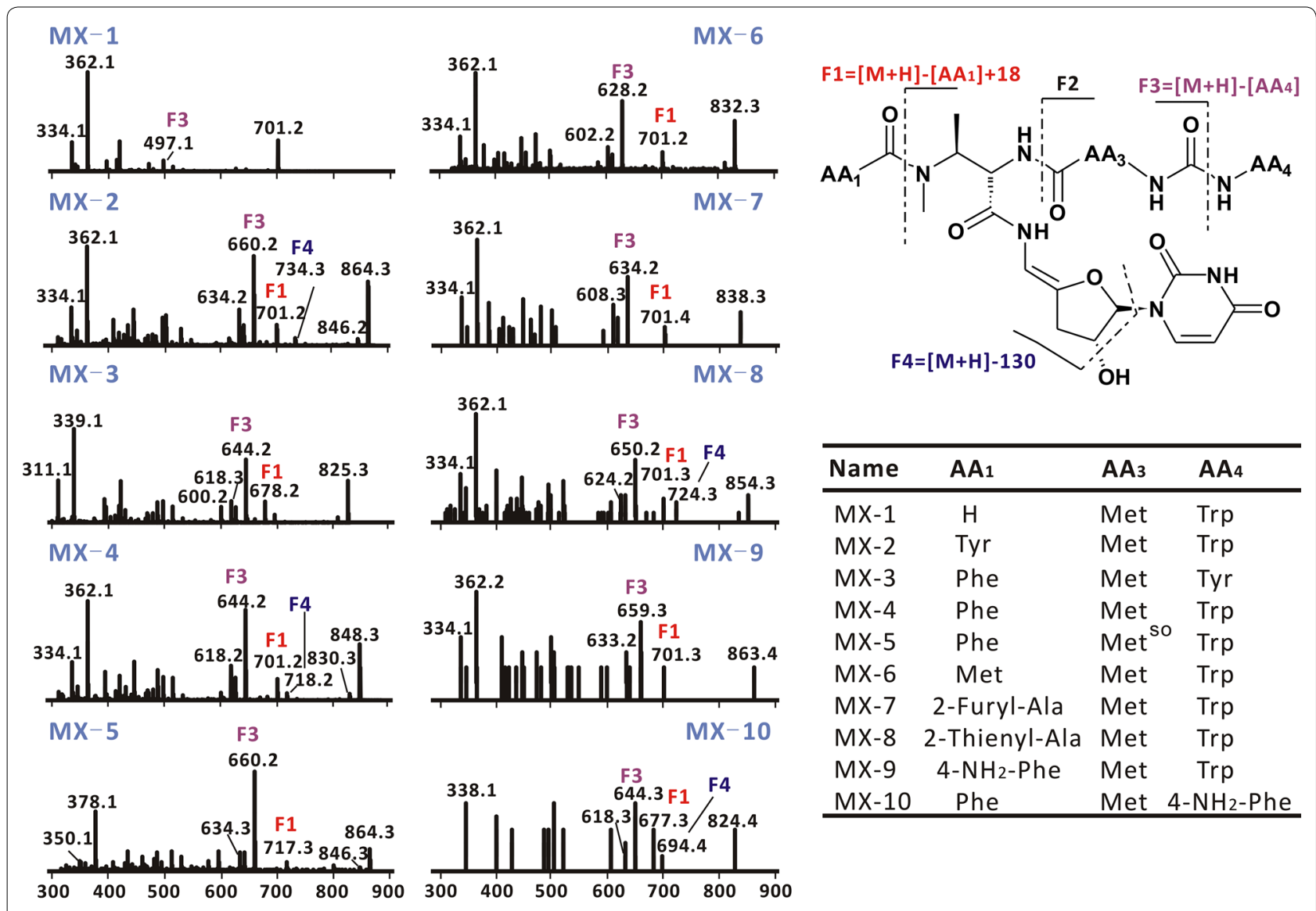

Fig. 4 (+)-ESI-MS/MS data and structures of ten new sansanmycin analogues

known sansanmycins by the virtue of bearing Tyr, Phe and Met at the N-terminus, which were firstly reported in the family of UPAs. The presence of the new sansanmycin analogues with various $\mathrm{N}$-terminal amino acids hinted that the NRPS responsible for the incorporation of the $\mathrm{N}$-terminal amino acid into the tetra-pseudopeptide backbone has moderate substrate promiscuity, suggesting that certain amounts of sansanmycin analogues might be able to be generated by mutational biosynthesis using ssaX deletion mutant $\mathrm{SS} / \mathrm{XKO}$.

\section{Generation of structurally diverse sansanmycin analogues using ssaX deletion mutant}

Initially twenty proteinogenic amino acids including Phe, Tyr and Met were used to probe the feasibility of mutasynthesis. The production of sansanmycin MX-2 which bears Tyr as its $\mathrm{N}$-terminus was nearly doubled when fed SS/XKO with Tyr (3 mM) (Fig. 3). Similarly, the production of sansanmycin MX-4 which bears Phe as its N-terminus was increased to two to three times when fed with Phe (3 mM) (Fig. 3). But the HPLC profile of the fermentation broth of ssaX deletion mutant fed with Met, as well as other proteinogenic amino acids $(3 \mathrm{mM})$ had no obvious changes (data not shown). This may be explained by the substrate preference of the NRPS, which preferred to select Phe or Tyr rather than any other proteinogenic amino acids. This result is consistent with the production level of sansanmycin analogues in SS/XKO. The improved production of sansanmycin MX-2 and MX-4 by feeding substrates Phe and Tyr suggested that mutasynthesis might be suitable to produce sansanmycin analogues with alternate $\mathrm{N}$-terminal substrate.

Incorporation of halogens into the molecules might exert a significant effect on their physicochemical properties of the products [26, 27]. Halogenated Phe had been fed to get sansanmycin analogues in the wild type strain by Xie et al. and they were preferably incorporated at the C-terminus $\left(\mathrm{AA}_{4}\right)$ [28]. In order to incorporate halogenated Phe into sansanmycin analogues at the $\mathrm{N}$-terminus efficiently, the ssaX deletion mutant SS/XKO was evaluated. Some new sansanmycin analogues were produced by SS/XKO and their structures were determined by ESIMS/MS. These sansanmycin analogues may be divided into three main groups (Fig. 6a). Compounds in the first 


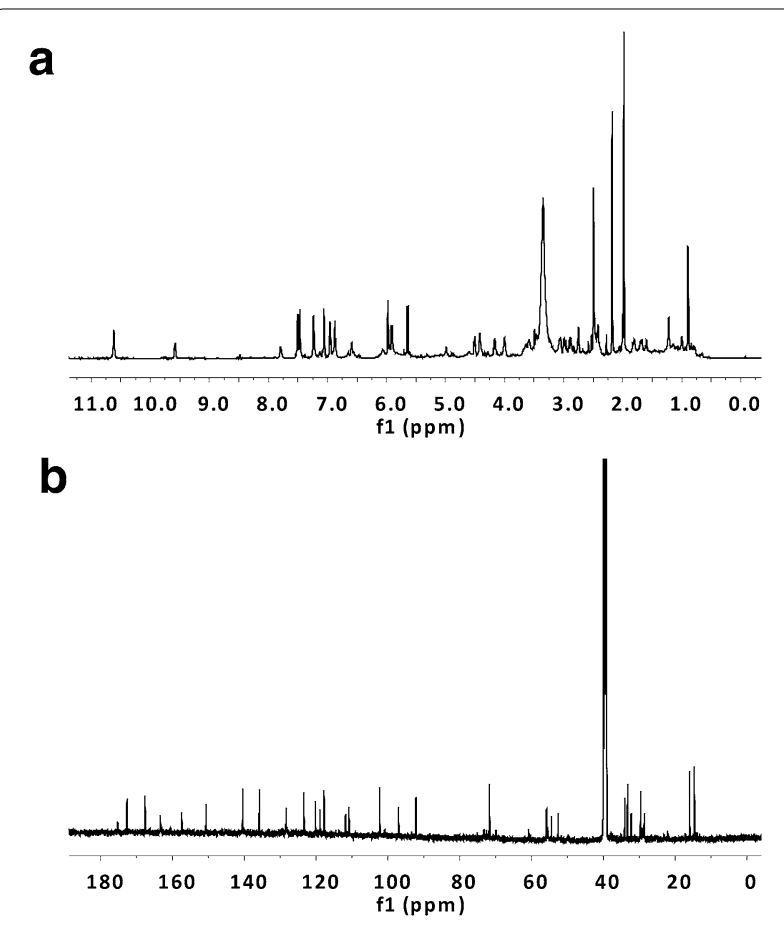

C

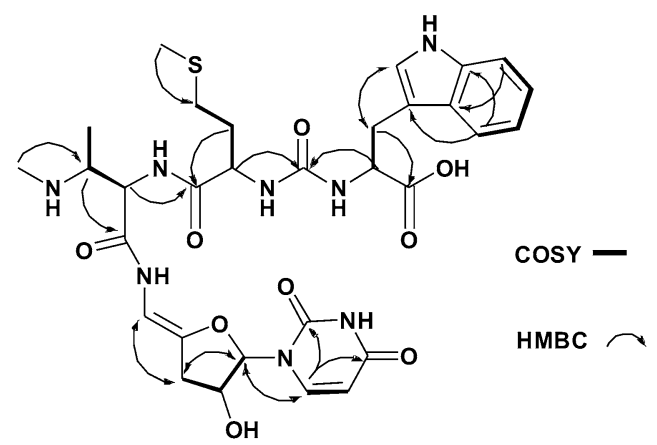

Fig. 5 Structural determination of sansanmycin MX-1. $\mathbf{a}^{1} \mathrm{H}$ NMR spectrum of sansanmycin MX-1 $\left(600 \mathrm{MHz}\right.$, DMSO- $\left.d_{6}\right)$ b ${ }^{13} \mathrm{C}$ NMR spectrum of sansanmycin MX-1 (150 MHz, DMSO- $\left.d_{6}\right)$ c Selected 2D NMR correlations for sansanmycin MX-1

and second groups were with halogenated Phe incorporated into the $\mathrm{N}$-terminus (e.g., compounds 1 and 2) or both the $\mathrm{N}$-terminus and the $\mathrm{C}$-terminus (e.g., compounds 3 and 4) respectively. The third group was with halogenated Phe incorporated into the C-terminus and with Phe at the N-terminus instead of $m$-Tyr (e.g., compounds 5 and 6). What's more, the production level of the analogues with halogenated Phe at the $\mathrm{N}$-terminus was increased remarkably compared with the wild type strain with the same growth curves. For example, when fed with 2-chloro- and 2-bromophenylalanines ( $3 \mathrm{mM})$, the production level of compound 1 and 2 was 11 - and 16-fold higher in SS/XKO respectively compared with the wild type strain (Fig. 6b). Similarly, compound 3 and 4 were increased 10- and 13-fold respectively (Fig. 6b). In a word, the $s s a X$ deletion mutant was a better host to get sansanmycin analogues with $\mathrm{N}$-terminus substitution, for its absence of the endogenous optimal substrate $m$-Tyr.

Motivated by the results of above feeding experiments and in order to expand the diversity of sansanmycins, more than 20 commercially available non-proteinogenic amino acid analogues were fed to SS/XKO (3 mM), including $\alpha$-amino acids and $\beta$-amino acids with diverse side chains, such as aliphatic groups, substituted aromatic phenyl groups, as well as aromatic and nonaromatic heterocyclic groups (Fig. 7). The results showed that $\beta$-amino acids detected here couldn't be incorporated into the peptidyl chain of sansanmycins. When feeding methyl substituted phenylalanines, the major products were the $\mathrm{C}$-terminal derivatives with $\mathrm{Phe}$ at the $\mathrm{N}$-terminus. When supplemented with para-amino-phenylalanine, two products with the fed precursor incorporated at the N- (MX-9) and the C-terminus (MX-10) respectively were obtained. Unexpectedly, two aromatic heterocyclic $\alpha$-amino acids, 2-furylalanine and 2-thienylalanine, can be incorporated into the $\mathrm{N}$-terminus to produce corresponding derivatives MX-7 and MX-8, respectively. The structures of MX-7-10 were determined by ESI-MS/MS (Fig. 4). MX-7, MX-8 and MX-9 showed the expected molecular weights and the same diagnostic fragment with sansanmycin $\mathrm{A}, \mathrm{m} / \mathrm{z} 701$ for the loss of the $\mathrm{N}$-terminal amino acid, suggesting the precursors added were incorporated into the N-terminus (Fig. 4). MX-10 displayed the same fragment with MX-4, m/z 644 corresponding to the loss of the $\mathrm{C}$-terminal amino acid, which suggested the $p$-amino-phenylalanine administrated was incorporated into the C-terminus (Fig. 4).

The yield of each sansanmycin derivative produced by mutasynthesis was estimated by the peak areas from HPLC (Table 1). The production level of sansanmycin MX-4 in SS/XKO without supplementation was considered as $100 \%$. When fed with $m$-Tyr, production of sansanmycin A was the highest. Surprisingly, the production level of MX-7 bearing 2-furylalanine was much higher than that of MX-2 bearing Tyr. All of these results suggested that other unnatural substrates might be acceptable for the mutant strain, especially aromatic $\alpha$-amino acids.

\section{Antibacterial activity and stability of novel sansanmycin analogues}

The obtained sansanmycin analogues MX-1-10 were tested for their antibacterial activity against different bacteria including gram-negative bacteria, gram-positive bacteria as well as $M$. tuberculosis $\mathrm{H}_{37} \mathrm{Rv}$ and clinically 
a<smiles>CSCCC(NC(=O)NC(Cc1c[nH]c2ccccc12)C(=O)O)C(=O)NC(C(=O)N/C=C1/CC(O)C(n2ccc(=O)[nH]c2=O)O1)C(C)N(C)C(=O)C(N)Cc1ccccc1Cl</smiles><smiles>CSCCC(NC(=O)NC(Cc1c[nH]c2ccccc12)C(=O)O)C(=O)NC(C(=O)N/C=C1/CC(O)C(n2ccc(=O)[nH]c2=O)O1)C(C)N(C)C(=O)C(N)Cc1ccccc1Br</smiles>

3<smiles>CSCCC(NC(=O)NC(Cc1ccccc1Cl)C(=O)O)C(=O)NC(C(=O)N/C=C1/CC(O)C(n2ccc(=O)[nH]c2=O)O1)C(C)N(C)C(=O)C(N)Cc1ccccc1Cl</smiles><smiles>CSCCC(NC(=O)NC(Cc1cccc(Br)c1)C(=O)O)C(=O)NC(C(=O)N/C=C1/CC(O)C(n2ccc(=O)[nH]c2=O)O1)C(C)N(C)C(=O)C(N)Cc1ccccc1</smiles>

4<smiles>CSCCC(NC(=O)NC(Cc1ccccc1Br)C(=O)O)C(=O)NC1C(=O)N/C=C2/CC(O)C(OC2n2ccc(=O)[nH]c2=O)C(=O)N(C(=O)C(N)Cc2ccccc2Br)C1C</smiles><smiles>CSCCC(NC(=O)NC(Cc1ccc(F)cc1)C(=O)O)C(=O)NC(C(=O)N/C=C1/CC(O)C(n2ccc(=O)[nH]c2=O)O1)C(C)N(C)C(=O)C(N)Cc1ccccc1</smiles>

b

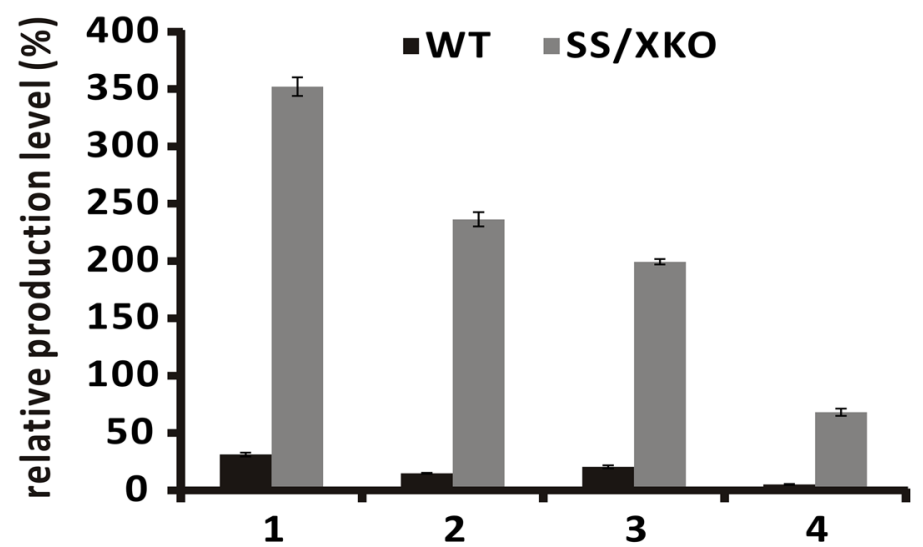

Fig. 6 The production of novel sansanmycin analogues. a The structures of novel compounds produced by ssaX deletion mutant SS/XKO fed with halogenated Phe. The final concentration of each exogenous substrate was $3 \mathrm{mM}$. The blue labeled parts represent the halogenated Phe incorporated into $\mathrm{N}$ - and/or C-terminus. b The relative production level of novel sansanmycin derivatives 1-4 in different strains. Chloramphenicol was added to the cultures as an extra standard 
<smiles>COc1ccc(C(N)CC(=O)O)cc1</smiles><smiles>N#Cc1ccc(C(N)CC(=O)O)cc1</smiles><smiles>NC(CC(=O)O)C(c1ccccc1)c1ccccc1</smiles><smiles>N[C@@H](CC(=O)O)Cc1ccc2ccccc2c1</smiles><smiles>C=CCC(N)CC(=O)O</smiles><smiles>N[C@@H](Cc1c(Cl)cccc1Cl)C(=O)O</smiles><smiles>Nc1ccc(CC(N)C(=O)O)cc1</smiles><smiles>N[C@@H](Cc1cccc([N+](=O)[O-])c1)C(=O)O</smiles><smiles>CC(C)(C)c1ccc(CC(N)C(=O)O)cc1</smiles><smiles>Cc1ccc(C[C@H](N)C(=O)O)cc1</smiles><smiles>Cc1cccc(CC(N)C(=O)O)c1</smiles><smiles>Cc1ccccc1CC(N)C(=O)O</smiles><smiles>N[C@@H](Cc1ccco1)C(=O)O</smiles><smiles>NC(Cc1cccs1)C(=O)O</smiles><smiles>N[C@@H](Cc1ccncc1)C(=O)O</smiles><smiles>NC1C=CC(C(=O)O)C1</smiles><smiles>O=C(O)C1C=CCN1</smiles><smiles>N[C@@H](CC1CC1)C(=O)O</smiles><smiles>NC1(C(=O)O)CCCC1</smiles>

Fig. 7 Structures of the commercially available non-proteinogenic amino acids fed to SS/XKO. Blue labeled substrates represent the non-proteinogenic amino acids successfully incorporated into the $\mathrm{N}$-terminus. The final concentration of each exogenous substrate was $3 \mathrm{mM}$

Table 1 Comparison of the relatively production level of sansanmycin analogues in ssaX deletion mutant SS/XKO

\begin{tabular}{lll}
\hline Fed substrate (3 $\mathbf{~ M})$ & $\begin{array}{l}\text { Sansanmycin } \\
\text { analogue }\end{array}$ & $\begin{array}{l}\text { Production } \\
\text { level (\%) }\end{array}$ \\
\hline - & sansanmycin MX-4 & 100 \\
m-Tyr & sansanmycin A & $\sim 350$ \\
Phe & sansanmycin MX-4 & $\sim 280$ \\
2-Cl/Br-Phe & compound 1, 2 & $240-270$ \\
2-Furyl-Ala & sansanmycin MX-7 & $\sim 240$ \\
Tyr & sansanmycin MX-2 & $\sim 80$ \\
2-Thienyl-Ala & sansanmycin MX-8 & $\sim 35$ \\
4- $-\mathrm{NH}_{2}$-Phe & sansanmycin MX-9 & $\sim 30$ \\
\hline
\end{tabular}

isolated MDR and XDR M. tuberculosis strains (Table 2). As expected, all the tested compounds (except MX-3) displayed different degrees of activity against $E$. coli $\triangle$ tolC mutant strain, which was consistent with previous results that UPAs can be exported by the AcrAB-TolC efflux system in E. coli [29]. Among them, sansanmycin MX-2 and MX-6 remained potency against $P$. aeruginosa equivalent to sansanmycin A. Interestingly, compound
MX-6 exhibited antibacterial activity against gram-positive B. subtilis, which was not found in natural UPAs. Sansanmycin MX-1, MX-2, MX-4, MX-6 and MX-7 showed potency against $M$. tuberculosis $\mathrm{H}_{37} \mathrm{Rv}$ comparable to sansanmycin A. More noticeably, these tested compounds exhibited equivalent potency against clinically isolated MDR and XDR strains. These results maybe lie in the fact that the target of UPAs is clinically unexploited.

During the early stage of drug development of sansanmycin A, we found that the structure of sansanmycin A was not stable at room temperature (Fig. 8a). As it is easier to get enough amount of sansanmycin MX-2 and MX-4, the stability test of sansanmycin A, MX-2 and MX-4 was performed in $\mathrm{KH}_{2} \mathrm{PO}_{4}$ buffer $(\mathrm{pH}$ 6.0) at room temperature. Under this condition, sansanmycin MX-2 and MX-4 kept mostly unchanged after 6 days of incubation whereas less than $10 \%$ sansanmycin A was remained (Fig. 8b), which suggested that sansanmycin MX-2 and MX-4 are more stable than sansanmycin A.

\section{Discussion}

During the past decade, considerable efforts have been made to exploit new UPA derivatives. Seventeen 
Table 2 Activities of sansanmycin analogues

\begin{tabular}{|c|c|c|c|c|c|c|c|}
\hline \multirow{3}{*}{$\begin{array}{l}\text { Compounds } \\
\text { (sansanmycin) }\end{array}$} & \multicolumn{7}{|l|}{ MIC $(\mu \mathrm{g} / \mathrm{ml})$} \\
\hline & \multirow[t]{2}{*}{ E. coli $\Delta$ tolC } & \multirow[t]{2}{*}{ P. aeruginosa 11} & \multirow[t]{2}{*}{ B. subtilis CMCC (B) 63501} & \multicolumn{4}{|c|}{ M. tuberculosis } \\
\hline & & & & $\mathrm{H}_{37} \mathrm{Rv}$ & FJ05189 & FJ05120 & FJ05195 \\
\hline$M X-1$ & 32 & $>128$ & $>128$ & 16 & 8 & 8 & 8 \\
\hline$M X-2$ & 2 & 32 & $>128$ & 16 & 16 & 8 & 8 \\
\hline$M X-3$ & $>128$ & $>128$ & 64 & & & & \\
\hline$M X-4$ & 8 & 64 & $>128$ & 8 & 16 & 8 & 16 \\
\hline$M X-5$ & 8 & $>128$ & $>128$ & & & & \\
\hline$M X-6$ & 4 & 32 & 16 & 8 & 8 & 8 & 8 \\
\hline$M X-7$ & 8 & 128 & $>128$ & 16 & 8 & 8 & 16 \\
\hline$M X-8$ & 8 & 128 & $>128$ & & & & \\
\hline MX-9 & 16 & 128 & $>128$ & & & & \\
\hline$M X-10$ & 64 & $>128$ & $>128$ & & & & \\
\hline A & 2 & 16 & $>128$ & 16 & 16 & 16 & 8 \\
\hline Streptomycin & 16 & 4 & 2 & 0.25 & $<0.06$ & $<0.06$ & $>128$ \\
\hline Isoniazid & & & & 0.125 & 8 & 16 & $>128$ \\
\hline Rifampicin & & & & $<0.06$ & 4 & $>128$ & $>128$ \\
\hline Ethambutol & & & & 0.5 & 2 & 0.5 & $>128$ \\
\hline
\end{tabular}

M. tuberculosis $\mathrm{H}_{37} \mathrm{Rv}$, standard and susceptible strain. FJ05189, FJ05120 and FJ05195 are clinical isolates of M. tuberculosis. FJ05189 and FJ05120, MDR strains, resistant to isoniazid and rifampicin; FJ05195, XDR strain, resistant to isoniazid, rifampicin, ethambutol, streptomycin, kanamycin and ofloxacin

sansanmycin analogues were semi-synthesized with sansanmycin A as the starting material, but most of them exhibited less anti-mycobacterial activity in comparison with parent natural product [30]. Strategy of precursordirected biosynthesis was employed to get pacidamycin analogues with modified C-terminal amino acid through feeding Trp derivatives [26]. The same strategy was also applied to sansanmycin-producing strain, resulting sansanmycin analogues with the C-terminus substituted by Phe derivatives [28]. Although some UPA analogues were produced, few of them had significantly improved antibacterial activity and/or physicochemical property. In the past 5 years, the biosynthetic pathways have been studied extensively at the genetic, enzymatic and regulatory levels [9-13], and bioengineering approaches are available to be used in producing novel UPA derivatives. In this work, mutational biosynthesis is employed by blocking the biosynthesis of $m$-Tyr and then feeding variety of alternative substrates to produce novel sansanmycin derivatives. This strategy is efficient to obtain novel sansanmycin analogues, creating a great structural diversity at the N-terminus.

In most of the reported UPAs, the N-terminus of the tetra-pseudopeptide $\left(\mathrm{AA}_{1}\right)$ was occupied by $m$-Tyr and its related bicyclic acids, except that pacidamycin D and $\mathrm{S}$ have an Ala at the N-terminus (Fig. 1). Two NRPSs responsible for selection of amino acid to incorporate into the $\mathrm{N}$-terminus of pacidamycin have been reported. PacU was demonstrated to specifically activate
Ala [5], and PacW was identified to activate $m$-Tyr [12]. In sansanmycin-producing strain, there are also two homologues of PacU, SsaU and SsaW, existed in sansanmycin biosynthetic gene cluster [11], but their amino acid sequences were almost exactly the same, with only one alteration of Lys to Arg [11]. Though they exhibited high overall homology with both PacU and PacW, the main residues in the binding pocket (specificity-conferring code) in the adenylation (A) domain [31, 32] were same with PacW but different from PacU. This is consistent with the fact that $m$-Tyr is the optimum substrate for the production of sansanmycin A in the wild type strain, and all the natural sansanmycin derivatives have $m$-Tyr and its related bicyclic acids at the N-terminus. When the biosynthesis of $m$-Tyr is blocked, Phe, Tyr, and small amount of Met may incorporate into the polypeptide of sansanmycin in ssaX deletion mutant, showing the potential of this mutant as a cell factory to expand the chemical diversity of $\mathrm{AA}_{1}$.

Various substrates including Phe, Tyr, halogenated Phe and other non-proteinogenic amino acids were fed to the ssaX deletion mutant and about 20 novel sansanmycin analogues with different $\mathrm{N}$-termini were produced according to the substrate promiscuity of the A domain of SsaU and SsaW. Ten of these compounds were purified and structurally determined by ESI-MS, ESI-MS/ MS (Fig. 4) and NMR. According to the production level of the compounds in ssaX deletion mutant fed with corresponding substrate (Table 1 ), the preference of the $\mathrm{A}$ 


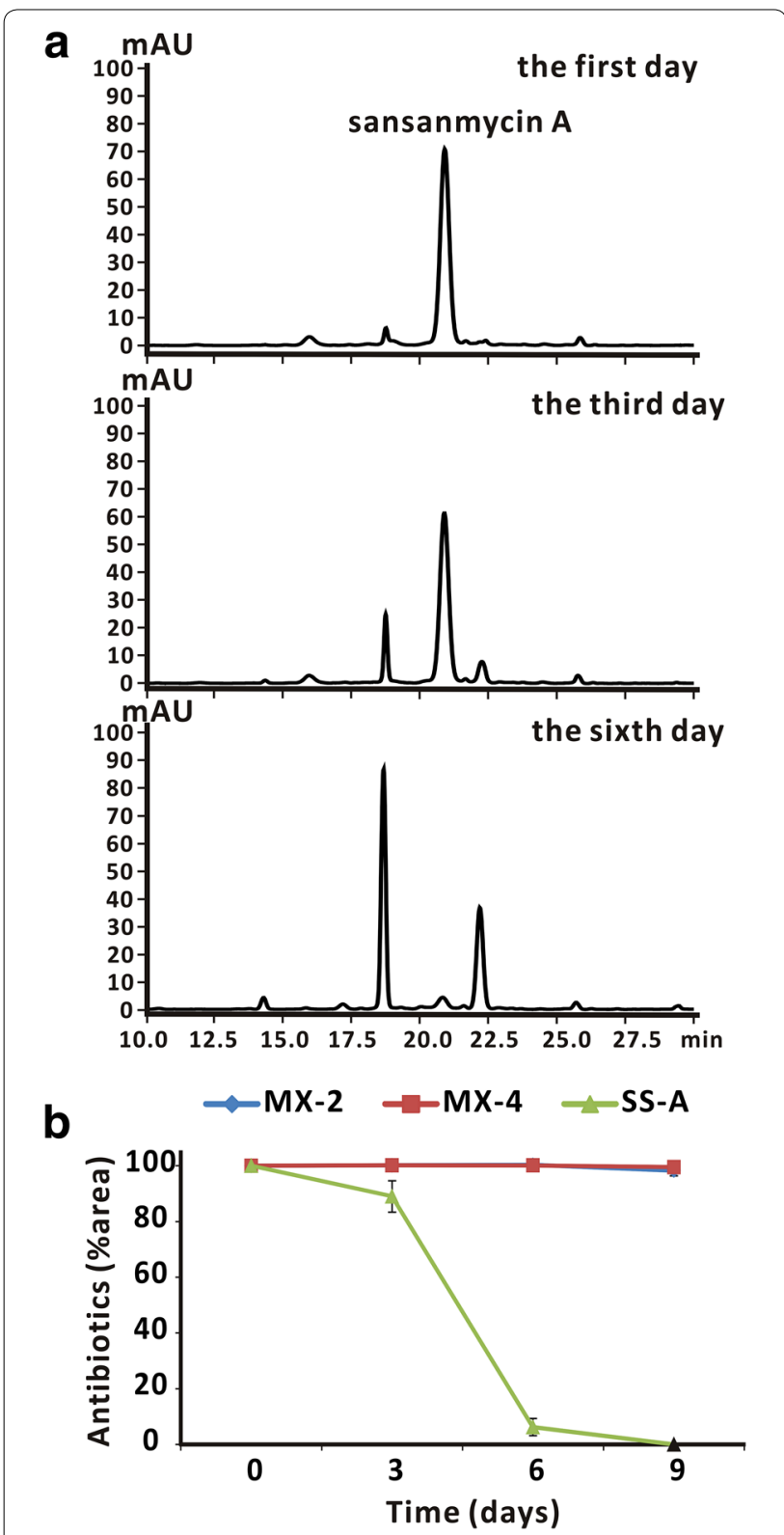

Fig. 8 Stability of sansanmycin $A, M X-2$ and MX-4. a HPLC analysis of sansanmycin $\mathrm{A}$ at indicated days in $\mathrm{KH}_{2} \mathrm{PO}_{4}$ buffer $(\mathrm{pH} 6.0$ ) at room temperature. $\mathbf{b}$ The changes of the level of sansanmycin $\mathrm{A}, \mathrm{MX}-2$ and MX-4 over time. All samples were analyzed by HPLC and quantified according to the areas of peaks. blue line, sansanmycin MX-2; red line, sansanmycin MX-4; green line, sansanmycin A

domain in charge of activating the sansanmycin $\mathrm{N}$-terminal amino acid is $m$-Tyr $>$ Phe $\geq$ ortho-halogenated phenylalanines $\geq 2$-furylalanine $>$ Tyr $>2$-thienylalanine $\geq p$-amino-phenylalanine $>$ Met. Out of our expectation, 2-furylalanine and 2-thienylalanine could be incorporated into the sansanmycin, suggesting the diversity of $\mathrm{AA}_{1}$ might be worth to explore further by trying more structurally diverse substrates. The compounds produced by mutasynthesis in this study retained the anti-TB activity of sansanmycin A in vitro, and more encouragingly, they showed similar activity to MDR and even XDR strains isolated from patients. Meanwhile, the stability of MX-2 and MX-4 was demonstrated to be greatly improved compared to sansanmycin A.

From the result of previous researches on the UPA derivatives of the wild type strains $[8,25]$ or by precursor-directed biosynthesis $[26,28]$, the A domain responsible for activating the $\mathrm{C}$-terminal amino acid $\left(\mathrm{AA}_{4}\right)$ has relatively high substrate promiscuity from Trp, Tyr to Phe and substituted Phe and Trp. On the other hand, the amino acid at the position of $\mathrm{AA}_{3}$ also can vary from Met and Ala [8] to Leu and Phe in sansanmycins [25, 33]. Now, together with the diversity at $\mathrm{AA}_{1}$ produced in this study, the combination of the variations in these three parts of the polypeptide backbone could be potentially expected that hundreds of new sansanmycin analogues might be obtained. Recently, crystal structure of Aquifex aeolicus MraY has been published [34], and the residues $\left(\mathrm{Asp}^{117}, \mathrm{Asp}^{118}, \mathrm{Asp}^{265}\right.$, and $\mathrm{His}^{324}$ ) important for the activity of MraY in the active site have been elucidated [34]. The structural information of MraY from A. aeolicus sets foundations for homologous modeling of MraY from M. tuberculosis [34, 35], which will facilitate the study on structure activity relationship (SAR) of novel chemically diverse UPA derivatives obtained by further rationale genetic engineering manipulation.

\section{Conclusion}

It is demonstrated that SsaX is responsible for the biosynthesis of $m$-Tyr in vivo by gene deletion and complementation and the sansanmycin production could be increased through the overexpression of ssaX. Six new sansanmycin analogues were purified and characterized in $s s a X$ deletion mutant, indicating the substrate flexibility of the responsible NRPS. The diversity of sansanmycin was further expanded by mutasynthesis, in which different types of substrates were fed to the culture of $s s a X$ deletion mutant. Totally ten compounds were purified, structurally identified and firstly reported. Five of them displayed anti-mycobacterial activity comparable to sansanmycin A and especially, they are active to MDR and even XDR M. tuberculosis clinical strains. In addition, sansanmycin MX-2 and MX-4 displayed significantly improved stability than sansanmycin A. These improved properties may promote the novel anti-TB drug investigation targeting a clinically unexploited target MraY. 


\section{Methods}

\section{Strains, plasmids and growth conditions}

The wild-type Streptomyces sp. SS strain, obtained from China Pharmaceutical Culture Collection (CPCC 200442), was used as a host strain for the propagation and disruption of genes, as described previously [11]. Streptomyces sp. SS and its derivatives were grown at $28{ }^{\circ} \mathrm{C}$ on solid S5 medium [36] for sporulation and in the liquid fermentation medium [1] for the production of sansanmycins. Mannitol soya flour (MS) agar [37] and liquid phage medium [38] were used for conjugation and isolation of genomic DNA respectively. Escherichia coli DH5 $\alpha$ was used as a host for general cloning experiments [39]. E. coli ET12567/pUZ8002 [40] was used for conjugal transfer according to the established protocol [37]. E. coli BW25113/pIJ790 was used as the host for Red/ETmediated recombination [21]. E. coli $\mathrm{DH} 5 \alpha$ containing the temperature sensitive FLP recombination plasmid BT340 was used as the host to remove the central part of the disruption cassette [21]. E. coli DH5 $\alpha$ and ET12567/ pUZ8002 were incubated in Luria-Bertani medium (LB) [39] at $37{ }^{\circ} \mathrm{C}$. E. coli BW25113/pIJ790 and DH5 $/$ BT340 were grown at $30{ }^{\circ} \mathrm{C}$ in LB medium. P. aeruginosa 11 , the indicator strain for the antibacterial bioassay of culture broth of different Streptomyces sp. SS strains [6], was grown on F403 agar [36]. E. coli $\Delta$ tolC mutant, P. aeruginosa 11, Bacillus subtilis CMCC (B) 63501, M. tuberculosis $\mathrm{H}_{37} \mathrm{Rv}$ and clinically isolated strains were used for testing antimicrobial activity for the compounds. When required, strains were incubated with apramycin (Am, $50 \mu \mathrm{g} / \mathrm{ml}$ ), ampicillin (Amp, $100 \mu \mathrm{g} / \mathrm{ml}$ ), spectinomycin (Spec, $50 \mu \mathrm{g} / \mathrm{ml})$, kanamycin $(\mathrm{Km}, 50 \mu \mathrm{g} / \mathrm{ml})$ and chloramphenicol $(\mathrm{Cm}, 30 \mu \mathrm{g} / \mathrm{ml})$. All the strains and plasmids used in this study are listed in Table 3.

\section{Construction and complementation of Streptomyces sp. SS ssaX mutant}

The ssaX in-frame deletion mutant SS/XKO was constructed by the $\lambda$-RED mediated PCR targeting method [21], using cosmid 13R-1 [11] covering ssaM-ssaV of sansanmycin biosynthetic gene cluster. In order to disrupt ssaX through homologous recombination, the minimal replicon of $\mathrm{SCP} 2 *$ of $13 \mathrm{R}-1$ was firstly replaced by ampicillin resistance marker bla, resulting 13R-1-SCP2KO. Then, a streptomycin resistance cassette (aadA gene) was amplified with primers PT-X-1 (5'-GCGGGAGGCCCCGCTGAACAGGGCCGCGAT GCTGTCGTCAT TCCGGGGATCCGTCGACC-3') and PT-X-2 (5'-GTCACCGACACCGCCTATGAGAA GCGCCGCGAGGAGATCTGTAGGCTGGAGCT GCTTC-3') including two 39-nt homologous extensions to sequences up- and downstream of the target ssaX gene. The cassette was introduced into E. coli BW25113/
pIJ790 to substitute ssaX on cosmid 13R-1-SCP2KO. The streptomycin resistance cassette on the correct recombinant cosmid was removed by FLP-recombinase in E. coli $\mathrm{DH} 5 \alpha / \mathrm{BT} 340$. The mutant cosmid 13R-1-SCP2KO-XKO was introduced into E. coli ET12567/pUZ8002 and then transferred into Streptomyces sp. SS by conjugation. Double-crossover exconjugants $\left(\mathrm{Am}^{\mathrm{s}}\right)$ were selected on MS agar with and without Am and confirmed by PCR using primers PT-X-7 (5'-TGAAGCCCGCCGCCTTTC-3') and PT-X-8 (5'-TCTGCCTTCCGCCTGACCAT- $\left.3^{\prime}\right)$ and southern blot hybridization using DIG Prime DNA Labeling and Detection Starter Kit I (for color detection with NBT/BCIP, Roche). The genomic DNAs were digested with $B a m \mathrm{HI}$ and hybridized with specific probes of ssaX deleted fragment amplified with primers SB-X-1 $\left(5^{\prime}\right.$-CTCGACCTCGTTCATGGAGT-3') and SB-X-2 (5'-AGTACGTCGACTGGGAGCAC-3') and the fragment downstream of $s s a X$ amplified with primers SB-X-3 (5'-AGAAACCACGATGCGAAATC-3') and SB-X-4 (5'-TGGATTTTTCGCTTCAAACC-3') respectively. The resulted $s s a X$ deletion mutant was designated SS/ $\mathrm{XKO}$.

For complementation analysis, complete ssaX coding region was amplified using primers pL-ssaX-F (5'-CGCATATGCAAGGGCATCGCGAC-3') and pL-ssaX-R (5'-ATAGGATCCTCAGCGCCGGGTG CC- $\left.3^{\prime}\right)$, and then cloned into the NdeI and BamHI sites of a pSET152-derived expression plasmid, pL646 [23], under the control of a strong constitutive promoter ermE $E^{*}$. The resulted expression vector $\mathrm{pL}-\mathrm{ssaX}$ was transferred into SS/XKO and Streptomyces sp. SS by conjugation to give the complementation strain and $s s a X$ overexpression strain respectively. The plasmid pSET152 [22] was transferred to SS/XKO and the wild type strain respectively as controls.

\section{Analysis of sansanmycin production}

Fermentation, isolation, and high-pressure liquid chromatography (HPLC) analysis of sansanmycins were carried out as described previously [1, 25]. In brief, pieces of well-grown agar cultures of different strains were firstly inoculated in fermentation medium and cultured at $28{ }^{\circ} \mathrm{C}$ for $48 \mathrm{~h}$ at $200 \mathrm{rpm}$. The obtained seed cultures were trans-inoculated into three parallel $100 \mathrm{ml}$ fermentation medium by $5 \%$ inoculation and grown at $28{ }^{\circ} \mathrm{C}$ for 5 days at $200 \mathrm{rpm}$. In the feeding test, each exogenous substrate was added to the fermentation medium to the final concentration of $3 \mathrm{mM}$. At indicated time points, five-milliliter cell cultures were collected by centrifugation and dried at $60{ }^{\circ} \mathrm{C}$ to constant weight for monitoring the growth curve. The obtained supernatants were analyzed for antibacterial activity and production of sansanmycins by bioassay and HPLC. Antibacterial 
Table 3 Strains and plasmids used in this study

\begin{tabular}{|c|c|c|}
\hline Strains/plasmids & Relevant characteristics & Reference \\
\hline \multicolumn{3}{|l|}{ Strains } \\
\hline Streptomyces sp. SS & Wild-type strain (sansanmycin-producing strain), CPCC 200442 & [1] \\
\hline $\mathrm{SS} / \mathrm{XKO}$ & Mutant of Streptomyces sp. SS with the in-frame deletion of ssaX & This study \\
\hline $\mathrm{SS} / \mathrm{XKO} / \mathrm{pL}-\mathrm{ssaX}$ & SS/XKO with the expression vector pL-ssaX, Am ${ }^{r}$ & This study \\
\hline SS/pL-ssaX & Streptomyces sp. SS with the expression vector pL-ssaX, $\mathrm{Am}^{r}$ & This study \\
\hline \multicolumn{3}{|l|}{ Escherichia coli } \\
\hline $\mathrm{DH} 5 \mathrm{a}$ & General cloning host & [39] \\
\hline ET12567/pUZ8002 & Donor strain for intergeneric conjugation between E. coli and Streptomyces, $\mathrm{Cm}^{r}, \mathrm{Km}^{r}$ & {$[40]$} \\
\hline BW25113/plJ790 & Strain for RED/ET-mediated recombination, $\mathrm{Cm}^{r}$ & [21] \\
\hline$\Delta$ tolC mutant & Strain for testing antimicrobial activity & [44] \\
\hline Pseudomonas aeruginosa 11 & Strain for sansanmycin bioassays & [6] \\
\hline Bacillus subtilis CMCC (B) 63501 & Strain for testing antimicrobial activity & \\
\hline Mycobacterium tuberculosis & Strain for testing antimicrobial activity & \\
\hline $\mathrm{H}_{37} \mathrm{RV}$ & Standard strain, susceptible to isoniazid and rifampicin & \\
\hline FJ05189 & Clinically isolated multi-drug-resistant strain, resistant to isoniazid and rifampicin & \\
\hline FJ05120 & Clinically isolated multi-drug-resistant strain, resistant to isoniazid and rifampicin & \\
\hline FJ05195 & $\begin{array}{l}\text { Clinically isolated extensive-drug-resistant strain, resistant to isoniazid, rifampicin, ethambutol, streptomy- } \\
\text { cin, kanamycin and ofloxacin }\end{array}$ & \\
\hline \multicolumn{3}{|l|}{ Plasmids } \\
\hline $13 R-1$ & $\begin{array}{l}\text { Cosmid based on vector pOJ446, containing the majority of sansanmycin biosynthetic gene } \\
\text { cluster ssaM-ssaV including ssaX }\end{array}$ & [11] \\
\hline 13R-1-SCP2KO & $\begin{array}{l}\text { Cosmid 13R-1 with the minimal replicon of } \mathrm{SCP}^{*} \text { replaced by ampicillin resistance marker bla, } \\
\mathrm{Amp}^{r_{1}} \mathrm{Am}^{r}\end{array}$ & This study \\
\hline 13R-1-SCP2KO-XKO & Cosmid 13R-1-SCP2KO with the in-frame deletion of ssaX, Ampr, Am ${ }^{r}$ & This study \\
\hline plJ779 & Vector used as the template for amplifying aadA cassette, Spec ${ }^{r}$ & {$[21]$} \\
\hline pSET152 & Streptomyces integrative vector, $\mathrm{Am}^{\mathrm{r}}$ & [22] \\
\hline pL646 & pSET152 derivative containing the constitutive promoter ermE* $\mathrm{p}, \mathrm{Am}^{r}$ & [36] \\
\hline pL-ssaX & pL646 derivative plasmid containing 843 bp complete coding region of ssaX & This study \\
\hline
\end{tabular}

$A m p^{r}$ ampicillin resistance, $A m^{r}$ apramycin resistance, $K m$ kanamycin resistance, $\mathrm{Cm}^{r}$ chloramphenicol resistance, Spec $^{r}$ spectinomycin resistance

activity was measured by cylinder plate method using P. aeruginosa 11 . For analyzing the expected analogues, the supernatant of fermentation broth was enriched by Sep-Pak $\mathrm{C}_{18}$ Classic Cartridge (Waters Associates, Milford, MA, USA), eluted with $60 \%$ methanol solution. The effluent was subjected to HPLC on an XBridge ${ }^{\mathrm{TM}} \mathrm{C}_{18}$ column $(4.6 \times 150 \mathrm{~mm}, 3.5 \mu \mathrm{m}$, Waters, Dublin, Ireland $)$ maintained at $40{ }^{\circ} \mathrm{C}$, with a gradient of $80: 200.1 \%(\mathrm{w} / \mathrm{v})$ $\left(\mathrm{NH}_{4}\right)_{2} \mathrm{CO}_{3}-\mathrm{MeOH}$ to $40: 60$ in $40 \mathrm{~min}$ as mobile phase at a flow rate of $1 \mathrm{ml} / \mathrm{min}$. Absorbance was monitored at $254 \mathrm{~nm}$. For the analysis of sansanmycin MX-3 and MX-6, the mobile phase was changed to $10: 90 \mathrm{MeOH}-$ $\mathrm{H}_{2} \mathrm{O}$ ( $\mathrm{pH}$ adjusted to 12.0 with $\mathrm{NH}_{3} \cdot \mathrm{H}_{2} \mathrm{O}$ ) in 40 min.

\section{Purification of sansanmycin analogues}

Isolation and purification of sansanmycin analogues was performed following the method of Xie et al. [25] with some modifications. Fifty liters of fermentation supernatant was obtained by centrifugation and then applied on a column of macroporous absorbant resin
4006. The active materials were eluted with $30 \%$ aqueous acetone. Then the effluent was applied on Toyopearl DEAE-Sephadex A25 eluted with Tris- $\mathrm{HCl}(20 \mathrm{mM}$, pH 8.5) plus $\mathrm{NaCl}$ and monitored by HPLC-UV. The concentration of $\mathrm{NaCl}$ was adjusted with different compounds from 0.01 to $0.05 \mathrm{M}$. The effluent containing target compounds was collected and further purified by preparative HPLC (YMC-Pack ODS-A $5 \mu \mathrm{m}, 250 \times 20 \mathrm{~mm}$ column, $0.1 \%(\mathrm{w} / \mathrm{v})\left(\mathrm{NH}_{4}\right)_{2} \mathrm{CO}_{3}-\mathrm{MeOH}$; flow rate, $5 \mathrm{ml} / \mathrm{min}$; UV detection at $254 \mathrm{~nm}$ and oven temperature at $40{ }^{\circ} \mathrm{C}$ ). The ratio of $0.1 \%(\mathrm{w} / \mathrm{v})\left(\mathrm{NH}_{4}\right)_{2} \mathrm{CO}_{3}$ and $\mathrm{MeOH}$ was dependent on different compounds. The structures of obtained compounds were determined using ESI-MS and ESI-MS/ MS (ThermoFisher LTQ Orbitrap XL mass spectrometer) as well as NMR [Varian Mercury 600 spectrometers, in dimethyl sulfoxide (DMSO)- $d_{6}$ ].

\section{Antibacterial assay}

The minimum inhibitory concentrations (MICs) for $M$. tuberculosis strains were determined by the microplate 
Alamar blue assay (MABA) [41]. All M. tuberculosis strains were grown on Middlebrook 7H9 medium supplemented with $0.2 \%(\mathrm{v} / \mathrm{v})$ glycerol and $10 \%(\mathrm{v} / \mathrm{v})$ OADC (oleic acid, albumin, dextrose, catalase) until the mid-log phase of growth at $37{ }^{\circ} \mathrm{C}$. The final suspension of bacteria cells were diluted in Middlebrook $7 \mathrm{H} 9$ medium to $10^{6} \mathrm{cfu} / \mathrm{ml}$. Initial compound dilutions were prepared in DMSO, and subsequent twofold dilutions were performed in $100 \mu \mathrm{l}$ of 7H9 (no Tween 80) in the microplates. Then, the MIC was measured in sterile 96-well plates with $100 \mu \mathrm{l}$ of the bacterial suspension and $100 \mu \mathrm{l}$ compound dilution per well. The MIC was defined as the lowest concentration of drug that prevented the color change of Alamar blue reagent from blue to pink. Rifampicin, isoniazid, ethambutol and streptomycin were used as controls.

The MICs for other bacterial strains were determined by a microdilution test following recommendations from the Clinical and Laboratory Standards Institute (CLSI, formerly NCCLS) [42]. The bacterial strains were grown on Mueller-Hinton broth (MHB) [43], and the final suspension of bacteria (in MHB medium) was adjusted to $10^{6}$ cells/ $\mathrm{ml}$. The dilutions of tested compounds were performed as method above with MHB medium instead. Then serial dilutions $(100 \mu \mathrm{l})$ were transferred to a $96-$ well plate in triplicate, and $100 \mu \mathrm{l}$ of the bacterial suspension was added to each well. After incubation at $37^{\circ} \mathrm{C}$ for $24 \mathrm{~h}$, the MIC was defined as the lowest concentration that inhibited the growth of the tested organism detected by visual observation. Streptomycin was used as the positive control.

\section{Stability determination of sansanmycin analogues}

To dissect the stability of sansanmycin A and other sansanmycin analogues, compounds were dissolved in $0.05 \mathrm{M} \mathrm{KH}_{2} \mathrm{PO}_{4}$ buffer (pH adjusted to 6.0 with $\mathrm{NaOH}$ ). All samples were incubated at $25^{\circ} \mathrm{C}$ for 9 days. Each sample has three parallel repeats. Residual analogues were analyzed by HPLC and quantified by the peak areas.

\section{Additional file}

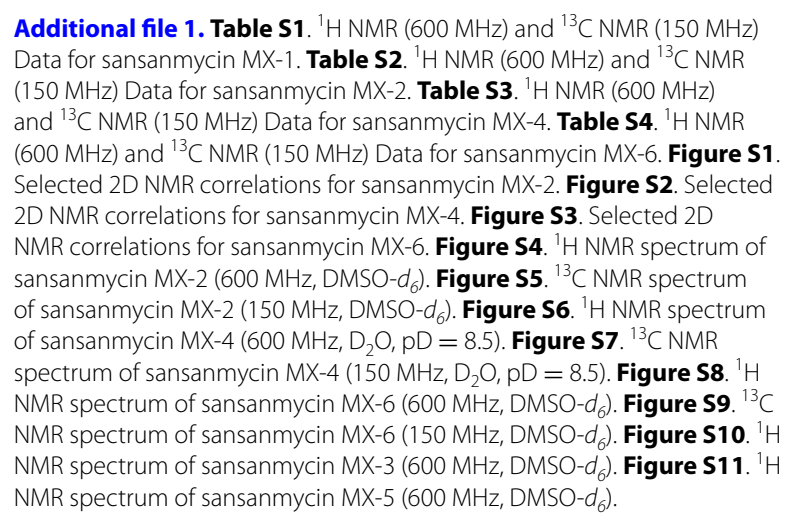

\section{Authors' contributions}

YS carried out experiments, analyzed the primary data and wrote the draft manuscript. ZJ and XL assisted with feeding experiments. NZ assisted with data analysis of MS and NMR, QC assisted with data analysis of MS, and QL designed ssaX mutant and assisted with relevant experiments. LW and SS assisted with testing antibacterial activity. YX supervised the chemical work in this study and revised the manuscript. BH supervised the whole research work and revised the manuscript. All authors read and approved the final manuscript.

\begin{abstract}
Acknowledgements
We thank Dr. Bertolt Gust (Pharmaceutical Institute, University of Tuebingen, Germany) for kindly providing Escherichia coli $\Delta$ tolC mutant strain. We thank Professor Kanglin Wan from the Chinese Center for Disease Control and Prevention (CCDC) for testing the anti-TB activity of compounds. This work was supported by the National Mega-Project for Innovative Drugs (2015ZX09102007-016, 2012ZX09301002-001-016 and 2014ZX09201001004-001) and the National Natural Science Foundation of China (81321004, $81402836,81273415,81302677$ and 31170042)
\end{abstract}

\section{Competing interests}

The authors declare that they have no competing interests.

Received: 3 February 2016 Accepted: 24 April 2016

Published online: 06 May 2016

References

1. Xie Y, Chen R, Si S, Sun C, Xu H. A new nucleosidyl-peptide antibiotic, sansanmycin. J Antibiot (Tokyo). 2007;60:158-61.

2. Chen RH, Buko AM, Whittern DN, McAlpine JB. Pacidamycins, a novel series of antibiotics with anti-Pseudomonas aeruginosa activity. II. Isolation and structural elucidation. J Antibiot (Tokyo). 1989;42:512-20.

3. Chatterjee S, Nadkarni SR, Vijayakumar EK, Patel MV, Ganguli BN, Fehlhaber HW, Vertesy L. Napsamycins, new Pseudomonas active antibiotics of the mureidomycin family from Streptomyces sp. HIL Y-82,11372. J Antibiot (Tokyo). 1994;47:595-8.

4. Isono F, Inukai M, Takahashi S, Haneishi T, Kinoshita T, Kuwano H. Mureidomycins A-D, novel peptidylnucleoside antibiotics with spheroplast forming activity II. Structural elucidation. J Antibiot (Tokyo). 1989;42:667-73.

5. Zhang W, Ostash B, Walsh CT. Identification of the biosynthetic gene cluster for the pacidamycin group of peptidyl nucleoside antibiotics. Proc Natl Acad Sci U S A. 2010;107:16828-33.

6. Xie $Y, X u H$, Si S, Sun $C$, Chen R. Sansanmycins B and C, new components of sansanmycins. J Antibiot (Tokyo). 2008;61:237-40.

7. World Health Organization. Global Tuberculosis Report 2015. Geneva: WHO Press; 2015

8. Winn M, Goss RJ, Kimura K, Bugg TD. Antimicrobial nucleoside antibiotics targeting cell wall assembly: recent advances in structure-function studies and nucleoside biosynthesis. Nat Prod Rep. 2010;27:279-304.

9. Rackham EJ, Gruschow S, Ragab AE, Dickens S, Goss RJ. Pacidamycin biosynthesis: identification and heterologous expression of the first uridyl peptide antibiotic gene cluster. ChemBioChem. 2010;11:1700-9.

10. Kaysser L, Tang X, Wemakor E, Sedding K, Hennig S, Siebenberg S, Gust B. Identification of a napsamycin biosynthesis gene cluster by genome mining. ChemBioChem. 2011;12:477-87.

11. Li Q, Wang L, Xie Y, Wang S, Chen R, Hong B. SsaA, a member of a novel class of transcriptional regulators, controls sansanmycin production in Streptomyces sp. strain SS through a feedback mechanism. J Bacteriol. 2013;195:2232-43.

12. Zhang W, Ntai I, Bolla ML, Malcolmson SJ, Kahne D, Kelleher NL, Walsh $\mathrm{CT}$. Nine enzymes are required for assembly of the pacidamycin group of peptidyl nucleoside antibiotics. J Am Chem Soc. 2011;133:5240-3.

13. Zhang W, Ntai I, Kelleher NL, Walsh CT. tRNA-dependent peptide bond formation by the transferase PacB in biosynthesis of the pacidamycin group of pentapeptidyl nucleoside antibiotics. Proc Natl Acad Sci U S A. 2011;108:12249-53. 
14. Qu X, Jiang N, Xu F, Shao L, Tang G, Wilkinson B, Liu W. Cloning, sequencing and characterization of the biosynthetic gene cluster of sanglifehrin A, a potent cyclophilin inhibitor. Mol BioSyst. 2011;7:852-61.

15. Zhang W, Ames BD, Walsh CT. Identification of phenylalanine 3-hydroxylase for meta-tyrosine biosynthesis. Biochemistry. 2011;50:5401-3.

16. Gentle C, Harrison S, Bugg TH. Structure-function studies on nucleoside antibiotic mureidomycin A: synthesis of 59-functionalised uridine models. J Chem Soc, Perkin Trans 1. 1999;1287-94.

17. Howard NI, Bugg TD. Synthesis and activity of 5'-uridinyl dipeptide analogues mimicking the amino terminal peptide chain of nucleoside antibiotic mureidomycin A. Bioorg Med Chem. 2003:11:3083-99.

18. Niu G, Tan H. Biosynthesis and regulation of secondary metabolites in microorganisms. Sci China Life Sci. 2013;56:581-3.

19. Feng C, Ling H, Du D, Zhang J, Niu G, Tan H. Novel nikkomycin analogues generated by mutasynthesis in Streptomyces ansochromogenes. Microb Cell Fact. 2014;13:59.

20. Song YN, Jiao RH, Zhang WJ, Zhao GY, Dou H, Jiang R, Zhang AH, Hou YY, Bi SF, Ge HM, Tan RX. New ansamycin derivatives generated by simultaneous mutasynthesis. Org Lett. 2015;17:556-9.

21. Gust B, Challis GL, Fowler K, Kieser T, Chater KF. PCR-targeted Streptomyces gene replacement identifies a protein domain needed for biosynthesis of the sesquiterpene soil odor geosmin. Proc Natl Acad Sci U S A 2003;100:1541-6.

22. Bierman M, Logan R, O'Brien K, Seno ET, Rao RN, Schoner BE. Plasmid cloning vectors for the conjugal transfer of DNA from Escherichia coli to Streptomyces spp. Gene. 1992;116:43-9.

23. Hong B, Phornphisutthimas S, Tilley E, Baumberg S, McDowall KJ. Streptomycin production by Streptomyces griseus can be modulated by a mechanism not associated with change in the adpA component of the A-factor cascade. Biotechnol Lett. 2007;29:57-64.

24. Usher JJ, Hughes DW, Lewis MA, Chiang SJ. Determination of the ratelimiting step(s) in the biosynthetic pathways leading to penicillin and cephalosporin. J Ind Microbiol. 1992;10:157-63.

25. Xie Y, Cai Q, Ren H, Wang L, Xu H, Hong B, Wu L, Chen R. NRPS substrate promiscuity leads to more potent antitubercular sansanmycin analogues. J Nat Prod. 2014;77:1744-8.

26. Gruschow S, Rackham EJ, Elkins B, Newill PL, Hill LM, Goss RJ. New pacidamycin antibiotics through precursor-directed biosynthesis. ChemBioChem. 2009;10:355-60.

27. Deb Roy A, Gruschow S, Cairns N, Goss RJ. Gene expression enabling synthetic diversification of natural products: chemogenetic generation of pacidamycin analogs. J Am Chem Soc. 2010;132:12243-5.

28. Zhang N, Liu L, Shan G, Cai Q, Lei X, Hong B, Wu L, Xie Y, Chen R. Precursor-directed biosynthesis of new sansanmycin analogues bearing para-substituted-phenylalanines with high yields. J Antibiot (Tokyo), in press.

29. Gotoh N, Murata T, Ozaki T, Kimura T, Kondo A, Nishino T. Intrinsic resistance of Escherichia coli to mureidomycin $\mathrm{A}$ and $\mathrm{C}$ due to expression of the multidrug efflux system AcrAB-TolC: comparison with the efflux systems of mureidomycin-susceptible Pseudomonas aeruginosa. J Infect Chemother. 2003;9:101-3.
30. Li YB, Xie YY, Du NN, Lu Y, Xu HZ, Wang B, Yu Y, Liu YX, Song DQ, Chen RX. Synthesis and in vitro antitubercular evaluation of novel sansanmycin derivatives. Bioorg Med Chem Lett. 2011;21:6804-7.

31. Stachelhaus T, Mootz HD, Marahiel MA. The specificity-conferring code of adenylation domains in nonribosomal peptide synthetases. Chem Biol. 1999;6:493-505.

32. Challis GL, Ravel J, Townsend CA. Predictive, structure-based model of amino acid recognition by nonribosomal peptide synthetase adenylation domains. Chem Biol. 2000;7:211-24.

33. Xie Y, Xu H, Sun C, Yu Y, Chen R. Two novel nucleosidyl-peptide antibiotics: sansanmycin $\mathrm{F}$ and $\mathrm{G}$ produced by Streptomyces sp SS. J Antibiot (Tokyo). 2010;63:143-6.

34. Chung BC, Zhao J, Gillespie RA, Kwon DY, Guan Z, Hong J, Zhou P, Lee SY. Crystal structure of MraY, an essential membrane enzyme for bacterial cell wall synthesis. Science. 2013;341:1012-6.

35. Fer MJ, Bouhss A, Patrao M, Le Corre L, Pietrancosta N, Amoroso A, Joris B, Mengin-Lecreulx D, Calvet-Vitale S, Gravier-Pelletier C. 5'-Methylenetriazole-substituted-aminoribosyl uridines as MraY inhibitors: synthesis, biological evaluation and molecular modeling. Org Biomol Chem. 2015;13:7193-222

36. Wang $L$, Hu Y, Zhang Y, Wang S, Cui Z, Bao Y, Jiang W, Hong B. Role of sgcR3 in positive regulation of enediyne antibiotic $C-1027$ production of Streptomyces globisporus C-1027. BMC Microbiol. 2009;9:14.

37. Kieser T, Bibb MJ, Buttner MJ, Chater KF, Hopwood DA. Practical Streptomyces Genetics. Norwich: John Innes Foundation; 2000.

38. Korn F, Weingartner B, Kutzner HJ. A study of twenty actinophages: morphology, serological relationship and host range. In: Freerksen E, Tarnok I, Thumin H, editors. Genetics of the Actinomycetales. New York: Gustav Fisher Verlag; 1978. p. 251-70.

39. Sambrook J, Russell DW. Molecular cloning: a laboratory manual. 3rd ed. Cold Spring Harbor Laboratory: Cold Spring Harbor; 2001.

40. Paget MS, Chamberlin L, Atrih A, Foster SJ, Buttner MJ. Evidence that the extracytoplasmic function sigma factor $\sigma^{\mathrm{E}}$ is required for normal cell wall structure in Streptomyces coelicolor A3(2). J Bacteriol. 1999;181:204-11.

41. Collins L, Franzblau SG. Microplate alamar blue assay versus BACTEC 460 system for high-throughput screening of compounds against Mycobacterium tuberculosis and Mycobacterium avium. Antimicrob Agents Chemother. 1997;41:1004-9.

42. CLSI. Methods for Dilution antimicrobial susceptibility tests for bacteria that grow aerobically: approved standard — 8th Edition. CLSI document M07-A8. Wayne: Clinical and laboratory standards institute; 2009.

43. Weissauer-Condon C, Engels I, Daschner FD. In vitro activity of four new quinolones in Mueller-Hinton broth and peritoneal dialysis fluid. Eur J Clin Microbiol. 1987;6:324-6.

44. Tang X, Gross M, Xie Y, Kulik A, Gust B. Identification of mureidomycin analogues and functional analysis of an $\mathrm{N}$-acetyltransferase in napsamycin biosynthesis. ChemBioChem. 2013;14:2248-55.

\section{Submit your next manuscript to BioMed Central and we will help you at every step:}

- We accept pre-submission inquiries

- Our selector tool helps you to find the most relevant journal

- We provide round the clock customer support

- Convenient online submission

- Thorough peer review

- Inclusion in PubMed and all major indexing services

- Maximum visibility for your research

Submit your manuscript at www.biomedcentral.com/submit 